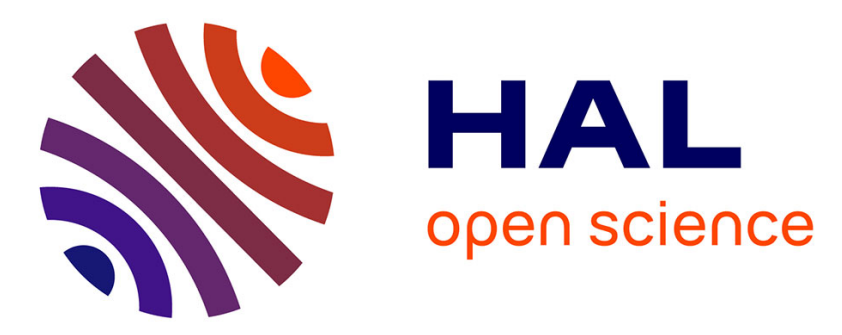

\title{
La sociologie sans les sociologues Assurer la présence du regard sociologique dans les organisations \\ Pascal Ughetto
}

\section{To cite this version:}

Pascal Ughetto. La sociologie sans les sociologues Assurer la présence du regard sociologique dans les organisations. Sociologies pratiques, 2016, Hors série 2, pp.137-145. 10.3917/sopr.hs02.0137 . hal-01343280

\section{HAL Id: hal-01343280 \\ https://hal-enpc.archives-ouvertes.fr/hal-01343280}

Submitted on 8 Jul 2016

HAL is a multi-disciplinary open access archive for the deposit and dissemination of scientific research documents, whether they are published or not. The documents may come from teaching and research institutions in France or abroad, or from public or private research centers.
L'archive ouverte pluridisciplinaire HAL, est destinée au dépôt et à la diffusion de documents scientifiques de niveau recherche, publiés ou non, émanant des établissements d'enseignement et de recherche français ou étrangers, des laboratoires publics ou privés. 


\title{
La sociologie sans les sociologues
}

\section{Assurer la présence du regard sociologique dans les organisations}

\author{
Pascal Ughetto \\ Université Paris-Est, Laboratoire Techniques, territoires, sociétés \\ In : Sociologies pratiques, $\mathrm{n}^{\circ} \mathrm{HS}, 2016, \mathrm{p} .137-145$.
}

Il y a un enjeu certain à vouloir affirmer la présence de la sociologie dans les organisations. Entreprises, administrations, associations ont plus que jamais besoin du regard favorisé par cette discipline. Mais ceux qui font profession de sociologues et, parmi eux, ceux qui pratiquent l'intervention en organisation ne portent qu'une partie de cet enjeu. La démultiplication de la présence de la sociologie doit plutôt compter sur des relais plus puissants que les seules interventions de ceux qui ont pour métier de faire de la sociologie : par exemple, les divers types d'intervenants dans les agences publiques (telle l'ANACT) et les cabinets de conseil ; mais également, pourquoi pas, toute personne issue d'une filière de sociologie et qui occupe une position professionnelle en entreprise ou dans une administration.

Le cas des étudiants inscrits dans des formations de niveau master à visée professionnelle (dans les domaines ressources humaines, développement urbain, communication, par exemple) mais qui exercent simultanément en entreprise dans le cadre de contrats d'apprentissage, de dispositifs d'alternance ou de stages doit donc recueillir notre attention. Formés en sociologie, ces étudiants font l'expérience, comme " alternants »-comme ils sont fréquemment nommés en entreprise -, de la difficile réception de l'approche sociologique. En cela, ils se heurtent à des obstacles qui sont très comparables à ceux de leurs enseignants, à ceci près que leur inscription dans une formation à dominante sociologique et, par conséquent, leur rapport à la discipline peuvent être l'effet des circonstances autant que d'un réel intérêt pour celle-ci et qu'ils n'ont encore ni une parfaite représentation de ce que devrait normalement être un rapport aux situations par la médiation de la sociologie ni les savoir-faire, les "ficelles du métier», totalement en main. Ces étudiants - la première partie le rappellera - se heurtent à des difficultés classiques. Le risque, cependant, pour la personne en formation est de les interpréter comme obligeant à choisir entre la posture et le questionnement sociologiques et les impératifs d'entreprise. Il est donc crucial - comme le montrera la deuxième partie - d'aider les futurs professionnels à faire quelque chose de cette tension.

1. Les difficultés de la réception de la sociologie en organisation : classiques pour son enseignant, nouvelles pour l'alternant

\section{1. Peu de demande de sociologie, ou des malentendus...}

L'apport de l'éclairage sociologique vis-à-vis des questions qui se posent en entreprise est plus que jamais à valoriser. Dans la période récente, les tensions ressenties par les salariés à l'égard 
de leur travail ont, par exemple, été l'occasion pour certains responsables d'entreprises de sentir confusément que des évolutions, dans l'activité, l'organisation, la relation des personnes au travail, avaient valeur d'énigme et méritaient de faire intervenir le sociologue pour mieux les analyser. " Ne pensez-vous pas qu'il y a un problème de perte du sens du travail ? ", peuvent-ils ainsi demander au sociologue quand ils le croisent. Cette demande traduit l'intuition que des dynamiques sont à l'œuvre, qui ne sont pas qu'individuelles. Mais la sociologie part avec des handicaps. En entreprise, on l'estime souvent concernée par les grandes évolutions de la société (on fait venir le sociologue pour faire une conférence sur les nouvelles générations, les violences ou le lien social) et des conclusions très générales. On l'appréhende également parfois comme fournissant des typologies de comportements (socio-styles...) directement utilisables dans les politiques marketing ou de communication et les formes de management. Autre possibilité : on la voit comme formulant des critiques et une dénonciation et comme méconnaissant les réalités économiques. Le paradoxe est donc que, si un intérêt pour la sociologie existe potentiellement dans les organisations, cette demande se heurte simultanément à de redoutables doutes à l'endroit de cette discipline. Ce que la sociologie est susceptible d'apporter n'est pas aisément identifié et les acteurs des organisations évoluent entre le scepticisme, voire le refus, et des attentes réduites à des réponses techniques et limitées, loin du domaine de compétence et de ce qui intéresse les sociologues.

L'apport du sociologue ne s'impose ainsi jamais avec la force de l'évidence, mais plutôt dans le cadre d'attentes et d'une demande dont l'expérience apprend qu'elles sont, quoi qu'il arrive, à gérer. On ne peut jamais considérer le contrat comme définitivement acquis et clairement connu et accepté de tous. C'est l'un des problèmes auxquels se heurteront les alternants et que connaissent les enseignants-chercheurs dans leurs propres recherches.

Dans un organisme HLM, je faisais récemment un entretien avec une responsable du développement des ressources humaines que je n'avais pas rencontrée comme interlocutrice dans la définition et négociation de l'objet de l'étude et de ses débouchés. La recherche portait sur l'essor des bâtiments visant la performance énergétique dans le logement social et les implications pour les métiers des personnels d'organismes et d'éventuels besoins en compétences. L'étude est née d'une démarche de ma part auprès d'interlocuteurs à l'Union sociale pour l'habitat (USH) pour développer un sujet susceptible d'intéresser le «mouvement HLM » tout en constituant un sujet d'enquête pour des étudiants de master et pour identifier des organismes acceptant d'y participer et d'accueillir ces étudiants pour l'enquête. Grâce à une réunion de travail avec un responsable du développement durable d'un organisme intéressé, le sujet finira par être défini comme visant à aider les organismes à mieux aborder la dimension métier et compétences qui, dans la transition vers un habitat plus performant au plan énergétique, reste mal maîtrisée par eux. Les deux organismes qui s'impliqueront en définitive dans la recherche ont été identifiés par l'USH dans le cadre de relations où ceux-ci se sont déjà tournés vers l'USH pour leur fournir des réponses aux nombreuses questions que soulève pour eux le développement de ces formes d'habitat.

Dans l'entretien, la responsable du développement des ressources humaines répond à des questions que je lui pose sur la façon dont l'organisme aurait ou non, d'ores et déjà, identifié des enjeux particuliers de métier et de compétences pour diverses catégories de personnels. L'échange la conduit à faire connaître sa représentation de la façon dont l'étude intervient dans ses efforts pour traiter ces questions : 
«Responsable ressources humaines: Comment vous dire? Quand on a commencé à travailler avec l'USH, on a posé la question en ces termes. "Ecoutez, on se lance dans une étude mais faut que ça débouche sur des solutions concrètes pour nous aider à trouver des [organismes de formation]" (...).

- Responsable développement durable (qui se trouve présent à ce moment de l'entretien): Pendant la journée de votre présence, nous, c'était un peu N. (nom de l'organisme HLM) qui était à l'initiative de cette étude et qui a demandé : "voilà, aidez-nous à voir quels sont nos besoins et identifier..." ; le deuxième axe, c'était "identifiez-nous des organismes compétents qui développent ces offres de formation".»

Le sociologue a alors la surprise d'apprendre que les attentes se révèlent comporter un pan d'intervention technique - l'identification des organismes de formation pertinents - qu'il n'avait jusqu'alors pas soupçonné et dont on imagine qu'il ne constitue pas son domaine de prédilection ni de compétence. L'échange, en tout cas, fait apparaître que, en cours même de démarche d'enquête - ne serait-ce que parce que chaque entretien oblige à préciser le cadre de la question traitée et à répondre aux acteurs mobilisés sur les débouchés de l'étude -, il y a à se confronter à une demande qui est mobile, voire inexistante, méfiante ou dirigée vers des attentes très techniques. La gestion de la demande doit négocier avec des acteurs qui estiment, souvent à juste titre, être engagés dans une réflexion suffisamment longue pour avoir, sans attendre le chercheur, analysé les problèmes qui se posent à eux et exploré des voies de solution. En quelque sorte, la demande adressée est alors : « aidez-nous sur les dernières étapes du chemin, à ce point où cela bloque».

C'est typiquement le problème auquel un alternant peut se trouver exposé et qui le conduira dans bien des cas à estimer qu'il y a un clivage entre la question de recherche et la question d'entreprise et à statuer au profit de cette dernière.

\subsection{Mais avancer, continuer, pour démontrer l'intérêt de la sociologie}

Avec l'expérience, cependant, son enseignant ne néglige pas qu'il existe des acteurs qui, sans être parfaitement avertis, identifient avec plus de pertinence l'intérêt à mobiliser le sociologue et qui font un travail de traduction. Et, surtout, il compte sur le fait que la démarche d'intervention (parfois même simplement le déroulement d'un entretien!) aide à avancer dans la perception par les acteurs de l'apport possible du sociologue : dans l'entretien évoqué, l'accumulation des questions faussement naïves finit par faire concevoir à la responsable du développement des ressources humaines que certaines manières d'envisager le problème n'ont pas été retenues et par la faire réfléchir, par exemple sur le fait de savoir si le besoin doit, au fond, nécessairement se chercher du côté de la formation.

En intervention, par conséquent, le chercheur aguerri tente de ne pas se laisser démonter par des réactions dubitatives ou hostiles et compte sur le temps (celui de l'entretien ou de l'intervention) pour renverser les opinions, apporter la preuve par l'exemple, faire concevoir ce que son intervention peut produire. Bref, il se fait confiance. C'est là - on le verra - une posture et une possibilité que les alternants ne font pas jouer de la même manière, eux qui se sentent volontiers pris entre deux instances d'affirmation du vrai et ne se voient pas en position de défendre le doute méthodologique de la sociologie face aux assertions des responsables d'entreprise. 
Le chercheur aguerri observe les appuis et alliés dont il dispose et joue également avec le travail que ces derniers effectuent, ne serait-ce que lorsqu'ils lui donnent des contacts et organisent des entretiens, opération qui les amène à exercer le rôle de traducteurs et à justifier l'utilité de coopérer. C'est une ressource dont il faut savoir jouer et que l'alternant ne perçoit pas forcément très bien, tandis que, à distance, son enseignant ne peut guère le lui faire comprendre. La démarche sociologique est connue d'acteurs des organisations parfois plus nombreux qu'on ne le pense; il faut savoir le mettre à profit. Dans les organismes HLM, il y a des directeurs généraux qui ont au moins eu des enseignements de sociologie ou d'ethnologie dans leur formation. Dans le domaine du travail social et de la politique de la Ville, les parcours de formation ont souvent conduit à assimiler les conclusions de la sociologie de P. Bourdieu. Chez bien des cadres des entreprises, la formation initiale ou continue a conduit à apprendre les bases de la sociologie des organisations. Il y a de la sociologie jusque dans les formations de gestion. Certes plus ou moins bien digérée au goût du sociologue patenté, la sociologie n'est pas radicalement méconnue et peut aider le sociologue à avoir des alliés.

Mais le chercheur sait qu'il n'a, quoi qu'il arrive, d'autre solution que de faire constater ce que sa discipline peut apporter et ce qu'elle est au fond. C'est en faisant qu'on fera concevoir. Dans l'intervalle, il lui faut « gérer ». En même temps, il faut aussi dire ce que cela peut produire ou ce que cela ne peut pas produire. C'est là encore une fois plus facile pour l'enseignant-chercheur qui peut délaisser la relation avec une organisation dont les acteurs sont, à son goût, trop peu réceptifs - que pour l'étudiant apprenti, bien obligé de faire avec son employeur.

\section{Diffuser la sociologie à travers la formation des étudiants}

Les étudiants qui se préparent à devenir des cadres d'entreprises et le font dans des formations à dominante sociologique pourront, dès qu'ils seront en emploi, être des relais pour faire exister le regard sociologique sans les sociologues. Mais beaucoup tient à la façon dont, durant leur formation en apprentissage, ils auront pu voir la sociologie, éventuellement comme suscitant des doutes chez leurs interlocuteurs d'entreprises, mais aussi comme une ressource qui finit par démontrer son apport. On pourrait en dire autant des doctorants sous convention Cifre $^{1}$ et des salariés en formation continue.

2.1. Des étudiants qui ne perçoivent pas nécessairement la ressource que constitue l'éclairage sociologique sur des questions de gestion

La formation à la sociologie débouche, à des niveaux comme le master, moins sur des programmes de formation à la recherche académique dans cette discipline (masters dits recherche) que sur des formations visant des débouchés professionnels (par exemple, former de futurs responsables de ressources humaines) où la sociologie n'est pas une fin en soi. Si l'enjeu est que, formés par des sociologues, ces étudiants transporteront ensuite avec eux, là où ils exerceront, le regard de la sociologie, il n'y a pas de garantie.

A cela, plusieurs obstacles. Les étudiants ne viennent pas obligatoirement à ces formations pour y être des sociologues appliquant leur savoir à des questions d'entreprises. L'étudiant issu d'une

${ }^{1}$ Cf. M. Berry, « Diriger des thèses de "terrain” », Gérer et comprendre, déc. 2000, p. 88-97. 
licence de sociologie peut attendre du programme qu'il lui permette de passer «enfin » de la théorie à de la pratique. Son condisciple se trouve peut-être, pour sa part, inscrit dans une formation dirigée par les sociologues mais en y voyant plutôt l'effet du hasard. Les masters professionnels peuvent comporter une variété de disciplines, en particulier la gestion. Or, pour les étudiants, il est souvent plus facile de se placer dans la pratique, normative, de la gestion que dans celle de la sociologie, davantage faite de mise à distance par la démarche compréhensive, de formes de relativisation des points de vue (la "guerre des dieux » de Max Weber) et de recul du moment de la préconisation. En termes de ressource pour l'exercice professionnel, la gestion est facilement perçue par les étudiants comme plus utile. Parce qu'ils cherchent par anticipation à occuper leur rôle professionnel, les étudiants peuvent préférer son regard, qui promet de savoir prendre la parole en manager pour dire ce qu'il faut faire. Au minimum, on les verra, malgré les indications qui peuvent leur être données sur la recherche bibliographique pertinente, retenir d'abord les écrits - ouvrages, en particulier - qui font fortement appel au style prescriptif. Plus généralement, ils peuvent avoir une préférence pour tout écrit privilégiant les sentences, tandis qu'un écrit plus analytique les laisse davantage dans l'embarras. Pour qui se confronte de manière assez nouvelle aux questions difficiles des entreprises - y compris un salarié en formation continue, pourtant plus familier de ces questions - toute rédaction donnant nettement à voir ce qu'elle enjoint aux acteurs aide en définitive davantage. A l'inverse, la sociologie ne laisse pas forcément facilement percevoir comment elle peut aider à exercer les rôles professionnels, en particulier managériaux. Les étudiants peuvent donc "passer à côté » de la ressource qu'ils pourraient rechercher dans cette discipline. Pour qu'elle exerce une influence par leur intermédiaire, il faut donc qu'elle puisse, en cours de formation, être découverte dans ce statut de ressource.

\subsection{Des obstacles bien difficiles à interpréter pour les apprentis}

Les sociologues en formation et étudiants exerçant en entreprise se heurtent frontalement aux mêmes problèmes que leurs enseignants, mais avec la difficulté d'une découverte tâtonnante de la mise en pratique du regard sociologique en organisation et donc peu de ficelles du métier ${ }^{2}$ à opposer aux obstacles, mais aussi la découverte de l'entreprise et le problème de la relation de subordination dans laquelle ils sont placés.

Les alternants se heurtent frontalement à la difficile réception de la démarche sociologique dans les organisations publiques ou privées. Aux conseils que leur donne leur enseignant, par exemple lorsqu'il s'agit de rédiger un mémoire de recherche autour de problèmes de l'entreprise appréhendés à l'occasion des missions d'apprentissage, l'étudiant répond souvent en opposant les difficultés sur lesquelles il bute dans l'entreprise : «Mon tuteur me dit que ce n'est pas cette question qui se pose ; selon lui, la véritable question est telle autre »; « mon tuteur ne veut pas que je fasse d'entretiens »; «mon tuteur demande surtout des préconisations ». Ces obstacles lui posent divers problèmes plus spécifiques qu'à son enseignant dans le cadre de ses recherches : a) le pouvoir hiérarchique qui s'impose à elle ou à lui, doublé par l'autorité avec laquelle parle son «tuteur» ou sa "tutrice», d'un ton qui n'appelle pas la discussion et en évoquant des empêchements dont l'alternant serait bien en peine d'apprécier l'éventuelle relativité ; b) l'arbitrage entre les régimes de vérité : la parole du hiérarchique est considérée par l'étudiant

${ }^{2}$ Cf. H. Becker, Les ficelles du métier, trad. fr., Paris, La Découverte, 2002, 352 p. 
comme exprimant le vrai ; et celle de son enseignant est sans doute à ses yeux un peu moins vraie, ne serait-ce que par le fait que cet enseignant en sait moins sur les réalités de l'entreprise d'accueil.

Fondamentalement, la confrontation au monde professionnel met à l'épreuve ce que l'étudiant pourrait avoir assimilé des bases du regard sociologique, à savoir que la réalité dans laquelle il se meut est construite : elle ne s'impose pas avec la nécessité que lui prêtent les propos des responsables et collègues dans l'entreprise, que l'alternant, prenant sa casquette de sociologue, devrait pourtant regarder également comme des «acteurs » à analyser. Toutes les apparences vont à l'encontre de cela : la force de la communication d'entreprise, l'autorité des rappels à l'ordre de la part des chefs, la conviction avec laquelle les collègues affirment que «c'est ainsi ». Si la gestion, à nouveau, a comme atout d'admettre l'idée de formuler des sentences, d'afficher des résultats qui servent les directives venues d'en haut, le sociologue, lui, doit opposer à toutes ces attitudes impressionnantes des acteurs de l'organisation qui l'accueille la pratique insistante de la naïveté (en substance, «je m'intéresse à ce que vous m'affirmez, mais je me permets de vous demander à nouveau pourquoi vous pensez ainsi »), la mise en évidence de la pluralité des points de vue, l'intérêt pour le caractère construit et sans doute fragile de la réalité. Il y a besoin de tout un savoir-faire pour tenir cette posture susceptible de s'attirer le mépris ou la réprobation. Il est difficile, dans une organisation, d'afficher des postures de doute sans donner l'impression de ne pas se placer dans le cadre des consignes hiérarchiques, voire de contribuer à la résistance au changement.

Tout cela est évidemment compliqué pour de jeunes gens dont le statut est précisément d'être encore en formation. Ce n'est d'ailleurs pas non plus très simple pour un salarié plus âgé en formation continue qui doit désapprendre à s'appuyer sur des certitudes pour agir et qui doit imposer des postures de questionnement susceptibles d'introduire le doute sur ses compétences professionnelles.

\subsection{Faire pratiquer la recherche-action}

Tout cela s'enseigne, notamment à travers des conseils donnés à l'étudiant en discutant, par exemple, de la définition d'un sujet et des stratégies d'enquête pour relier des problèmes d'entreprise et une problématique sociologique. Mais l'enseignant doit piloter à distance. Il propose, suggère, mais dépend de ce que l'étudiant lui rapporte des obstacles et occasions, sans pouvoir apprécier s'il pourrait y avoir des façons de procéder ou de dire qui relativiseraient considérablement ce que l'étudiant a tendance à présenter comme une difficulté rédhibitoire. Il y a donc des limites. Autant que possible, par conséquent, il faut faire voir, faire toucher du doigt. Si un point crucial est d'expliquer en quoi consiste le regard sociologique (en veillant à ne pas oublier que ce regard a quelque chose de déroutant pour celui qui le rencontre pour la première fois et qu'il faut du temps pour s'y acclimater), il est indispensable de faire pratiquer la recherche-action. Sur des terrains qui ne sont pas les lieux d'apprentissage des étudiants et où ils seront moins inquiets de ne pas satisfaire leur employeur, il s'agit de leur faire voir comment on prend au sérieux une question adressée par une organisation, mais aussi comment on la traite comme étant formulée par des acteurs, susceptible d'être confrontée à des retours du terrain (propos d'interviewés, observations) qui ne s'alignent pas immédiatement dessus pour donner une réponse immédiate. Même si le volume horaire de l'enseignement ne permet pas de 
leur faire traiter la demande de bout en bout et si leur enseignant prend en main une partie de l'analyse, il est au moins possible de mettre en scène grâce à cela l'attitude du sociologue qui s'attelle à résoudre la question posée et se tient cependant prêt à justifier des reformulations, ou qui fait réfléchir à la manière dont une analyse rend audible ou difficilement tolérable la restitution de points de vue divergents.

La recherche-action se révèle sans doute constituer le meilleur moyen de faire comprendre, mieux que des explications abstraites, en quoi consiste une approche sociologique des questions d'entreprises; elle permet de faire passer des messages (par exemple, de prudence dans l'interprétation des dires recueillis) qui trouvent leur efficacité dans le fait d'être directement situés ; elle permet de faire découvrir une variété d'acteurs et de groupes professionnels que des étudiants en formation initiale connaissent généralement mal et, surtout, d'apprendre à ces étudiants qui exerceront souvent dans les bureaux des sièges des organisations à aller au contact des réalités professionnelles et de leurs technicités; elle permet de constituer des compétences (de mise en problématique, de recueil de données...) vis-à-vis desquelles, spontanément, des étudiants ne se destinant pas à devenir sociologues pourraient ne pas être réceptifs.

Dans le cas évoqué dans la première partie, un sujet et un accès au terrain ont été négociés avant de le confier aux étudiants. Mais expliquer à ces derniers le travail qui va désormais devoir être le leur est l'occasion de leur expliquer les conditions de cette négociation, les ambiguïtés et incertitudes dont on hérite, parce qu'il faut bien leur dire à quelles difficultés de réalisation de leur travail de terrain ils vont éventuellement se heurter. C'est surtout l'occasion de leur faire voir deux choses.

Tout d'abord, que le travail de terrain ne ramène généralement rien qui soit directement en accord avec les besoins de l'entreprise directement exprimés. Les constructions nouvelles et équipements qui visent la performance énergétique transforment-ils les métiers des personnels des organismes HLM et ces personnels disent-ils qu'ils ont besoin d'être formés et sur quoi ? Les étudiants ont été désappointés en revenant de la première vague d'entretiens avec le résultat que la plupart des représentants des métiers leur disaient ne pas voir grande évolution. Si cette question avait été celle qui leur avait été confiée dans leur entreprise d'accueil, cela aurait peutêtre conduit à faire avorter toute réflexion. Le tuteur aurait affirmé : «l'étude de mon étudiant(e) montre qu'il n'y a pas d'effet et pas de besoins de montée en compétence ", et cela aurait été pris comme un fait prouvé, fermant définitivement le sujet. Dans la recherche-action encadrée, l'enseignant fait travailler les résultats plus en profondeur pour mieux faire réfléchir à l'interprétation du résultat apparent (est-ce parce que les intéressés n'ont pas encore été suffisamment touchés pour voir ce que cela pourrait changer dans leur métier, ou parce qu'il n'ont pas pris conscience de ce que cela pourrait changer faute d'avoir connu des expériences malheureuses sur les nouveaux bâtiments, susceptibles de les amener à concevoir l'enjeu de nouvelles pratiques professionnelles ?). Au bout du compte, la recherche en viendra à conclure que les évolutions technologiques considérées opposent aux divers métiers des problèmes qui ne sont pas d'une nature radicalement nouvelle mais donnent une intensité nouvelle à des enjeux (de qualité des coordinations, de dialogue entre métiers...) déjà présents dans d'autres évolutions du secteur depuis plusieurs années. Elle conduit aussi au message à l'intention des directions des organismes que le problème n'est que secondairement celui de besoins de formations (et donc encore moins d'identification des organismes de formation!) qu'un problème d'organisation et de qualité de l'animation managériale de l'apprentissage collectif. 
Deuxième chose, c'est l'occasion de faire voir aux étudiants comment les affirmations des membres de la hiérarchie peuvent être traitées comme des propos d'acteurs et utilisées dans le diagnostic et que ceux-ci doivent se voir conférer par la démarche d'enquête un autre statut que celui d'informateurs en dehors de la situation.

Même si, faute de temps, l'enseignant concentre les étudiants sur la réalisation d'entretiens et d'observations, l'animation d'une discussion simplement sur ce qu'ils en retirent, les impressions qui ont été les leurs, permet de faire travailler, par exemple, sur la possibilité de se déplacer de grilles de lecture spontanées (Madame $\mathrm{X}$ a bien dit que, et donc Monsieur $\mathrm{Y}$ a tort quand il dit l'inverse) vers d'autres plus analytiques (la réalité est constituée de gens qui, comme Madame X, estiment que, et d'autres, comme Monsieur Y, qui, au contraire, pensent que...). Cela permet aussi de leur montrer qu'ils ne perdent rien à cette mise en forme des résultats - moins binaire ou tranchée, plus nuancée - et qu'elle augmente au contraire leurs chances de jouer un rôle consistant à aider les acteurs à concevoir des solutions de conciliation des points de vue là où ils agissent peut-être en entretenant des clivages et des incompréhensions. La restitution des résultats devant les responsables de l'entreprise peut se faire en la confiant aux étudiants ou en les laissant simples observateurs. Cette seconde option n'est pas nécessairement de moindre intérêt car elle se prête moins à voir les responsables d'entreprise pratiquer l'indulgence qui les gagne volontiers quand les étudiants exposent scolairement devant eux.

$\mathrm{Au}$ fond, la recherche-action est surtout l'occasion de faire voir aux étudiants comment leurs enseignants pratiquent le métier lors d'une intervention et de compter ainsi sur des formes d'appropriation. On peut alors espérer que les étudiants ainsi formés transporteront plus facilement le regard sociologique dans leur entreprise d'accueil et dans leurs futurs emplois.

\begin{abstract}
Résumé
L'article s'intéresse aux étudiants inscrits dans des formations de niveau master à visée professionnelle et qui exercent simultanément en entreprise dans le cadre de contrats en alternance. Alors qu'ils font l'expérience de la difficile réception de l'approche sociologique, comment les aider à voir la sociologie comme leur offrant des ressources pour répondre à des enjeux d'analyse et de propositions d'action là où ils voient d'abord les malentendus, incompréhensions, refus auxquels ils se heurtent? La recherche-action est ici présentée comme constituant un moyen intéressant de faire comprendre comment leur discipline leur permet de répondre aux sollicitations dont ils sont l'objet.
\end{abstract}

Mots-clés

Recherche-action ; sociologie ; entreprise ; étudiants ; master. 